\title{
Cortical $\beta$-Amyloid in Older Adults Is Associated with Multidomain Interventions with and without Omega 3 Polyunsaturated Fatty Acid Supplementation
}

\author{
C. Hooper ${ }^{1}$, N. Coley ${ }^{2,3}$, P. De Souto Barreto ${ }^{1,2}$, P. Payoux ${ }^{4,5}$, A.S. Salabert ${ }^{4,5}$, S. Andrieu ${ }^{2,3}$, M. Weiner ${ }^{6}$, \\ B. Vellas ${ }^{1,2}$ for the MAPT/DSA study group
} 1. Gérontopôle, Department of Geriatrics, CHU Toulouse, Purpan University Hospital, Toulouse, France; 2. UMR1027, Université de Toulouse, UPS, INSERM, Toulouse,
France; 3. Department of Epidemiology and Public Health, CHU Toulouse, Toulouse, France; 4. UMR 1214, Toulouse Neuroimaging Center, University of Toulouse III,
Toulouse, France; 5 . Department of Nuclear Medicine, University Hospital of Toulouse (CHU-Toulouse), Toulouse, France; 6 . University of California San Francisco,
School of Medicine, 4150 Clement Street, San Francisco, California. USA.

Corresponding Author: Claudie Hooper, Gérontopôle, Department of Geriatrics, CHU Toulouse, Purpan University Hospital, Toulouse, France , Tel : +33 (5) 6177 64 25, Fax : +33 (5) 61776475 claudie28@yahoo.com

J Prev Alz Dis 2020;2(7):128-134

Published online February 5, 2020, http:/ / dx.doi.org/10.14283/jpad.2020.4

\begin{abstract}
Multidomain lifestyle interventions (including combinations of physical exercise, cognitive training and nutritional guidance) are attracting increasing research attention for reducing the risk of Alzheimer's disease (AD). Here we examined for the first time the cross-sectional relationship between cortical $\beta$-amyloid $(\mathrm{A} \beta)$ and multidomain lifestyle interventions (nutritional and exercise counselling and cognitive training), omega 3 polyunsaturated fatty acid (n-3 PUFA) supplementation or their combination in 269 participants of the Multidomain Alzheimer Preventive Trial (MAPT). In adjusted multiple linear regression models, compared to the control group (receiving placebo alone), cortical $A \beta$, measured once during follow-up (mean $512.7 \pm 249.6$ days post-baseline), was significantly lower in the groups receiving multidomain lifestyle intervention + placebo (mean difference, $-0.088,95 \% \mathrm{CI},-0.148,-0.029, \mathrm{p}=$ $0.004)$ or multidomain lifestyle intervention $+n-3$ PUFA $(-0.100$, $95 \% \mathrm{CI},-0.160,-0.041, \mathrm{p}=0.001)$, but there was no difference in the n-3 PUFA supplementation alone group (-0.011, $95 \% \mathrm{CI}$, $-0.072,0.051, p=0.729)$. Secondary analysis provided mixed results. Our findings suggest that multidomain interventions both with and without n-3 PUFA supplementation might be associated with lower cerebral $A \beta$. Future trials should investigate if such multidomain lifestyle interventions are causally associated with a reduction or the prevention of the accumulation of cerebral $\mathrm{A} \beta$.
\end{abstract}

Key words: Multidomain lifestyle intervention, $\beta$-amyloid, physical activity, cognitive activity, nutrition, Alzheimer's disease.

\section{Introduction}

E vidence suggests that the individual components of multidomain lifestyle interventions, including cognitive activity $(1,2)$, physical activity $(3,4)$ and nutrition $(5,6)$ are associated with reduced cerebral $\beta$-amyloid $(A \beta)$. Physical activity has been cross-sectionally associated with reduced central A $\beta$ in cognitively normal older adults (4) as well as in autosomal dominant (early onset familial) cases of Alzheimer's disease (AD) $(3,7)$. A less active lifestyle has been associated with more cerebral $A \beta$ in apolipoprotein E (ApoE) $\varepsilon 4$ carriers (8) and the association of physical activity with reduced cerebral $A \beta$ appears to be more prominent in ApoE $\varepsilon 4$ carriers $(4,9)$. Furthermore, longterm treadmill exercise reduces $A \beta$ in murine models of AD possibly through reduced amyloidogenic-cleavage $(10,11)$ and / or increased $A \beta$ degradation (10).

Lifetime cognitive activity has been cross-sectionally associated with reduced cerebral $A \beta$ in human subjects (1) and in another cross-sectional study it was shown that $A \beta$ was diminished in ApoE $\varepsilon 4$ carriers that reported higher cognitive activity over the course of life (2). Moreover, lifetime intellectual enrichment (high education, high midlife cognitive activity) has been associated with lower cortical $A \beta$ deposition longitudinally in ApoE $\varepsilon 4$ carriers (12). However, there is in vitro and animal data to indicate that neural activity increases the secretion of $A \beta(13,14)$, which might lead to enhanced deposition if clearance mechanisms failed. Nevertheless, consistent with our hypothesis, transgenic $A \beta$-expressing mice exposed to enriched environments deposit less $A \beta$ than control animals (15).

In terms of nutrition and $A \beta$, increased cerebral $A \beta$ has been associated with a high glycaemic diet (16) and a lack of adherence to a Mediterranean style diet (17) and vitamin B12 as well as vitamin D (5) have been inversely associated with cerebral $A \beta$. Cell culture and animal models suggest that docosahexaenoic acid (DHA), the predominant omega (n-3) polyunsaturated fatty acid (PUFA) in the brain, might reduce $\mathrm{A} \beta$ production (18-20) and serum DHA has been inversely associated with brain $\mathrm{A} \beta$ cross-sectionally in older adults (21). To the contrary, however, we have previously reported that erythrocyte membrane DHA, eicosapentaenoic acid (EPA) as well as total n-3 PUFA were not cross-sectionally associated with cortical $A \beta$ in participants of the placebo group of Multidomain Alzheimer Preventive Trial (MAPT) (22). 
Using similar multidomain lifestyle interventions to those used in MAPT (nutritional and exercise counselling and cognitive training), other trials have explored the effects of multidomain interventions targeting a healthier lifestyle on cognitive function in older adults (23-26). However, to the best of our knowledge no information is available on the relationship between multidomain lifestyle interventions and cerebral $A \beta$ burden. Hence we explored the cross-sectional relationship between cortical $\mathrm{A} \beta$ and multidomain lifestyle interventions, n-3 PUFA supplementation or their combination in 269 participants of the MAPT trial who underwent voluntary $\left[{ }^{18} \mathrm{~F}\right]$ florbetapir positron emission tomography (PET). We hypothesised that multidomain lifestyle intervention might be associated with reduced cerebral $A \beta$ and that this association might be potentiated by $n-3$ PUFA supplementation.

\section{Methods}

\section{The Multidomain Alzheimer Preventive Trial (MAPT) and ethical approval}

Data were obtained from a $\left[{ }^{18} \mathrm{~F}\right]$ florbetapir PET study carried out as an ancillary project to MAPT (registration: NCT00672685), a large multicentre, phase III, randomized, placebo-controlled trial (RCT) which has already been described in detail (26). MAPT subjects $(n=1680)$ were randomized to one of the four following arms: n-3 PUFA supplementation alone, multidomain lifestyle intervention (involving nutritional and exercise counselling and cognitive training) + placebo, multidomain lifestyle intervention $+n-3$ PUFA supplementation, or placebo alone (control group). Both MAPT and the PET sub-study were approved by the ethics committee in Toulouse (CPP SOOM II) and written consent was obtained from all participants.

\section{Participants}

At inclusion, subjects were community-dwelling men and women without dementia, aged $\geq 70$ years, and who met at least one of the following criteria: spontaneous memory complaints, limitation in executing $\geq 1$ Instrumental Activity of Daily Living, or slow gait speed $(<0.8$ meters/sec). Participants of the study described here were 269 individuals who had data on cortical $\mathrm{A} \beta$ (excluding two participants who developed dementia as assessed at the clinical evaluation closest to PET-scan (Clinical Dementia Rating $(C D R) \geq 1)$ ). MAPT participants who were not assessed for cerebral $A \beta$ (n $=1408$ ) were similar to the participants in the PET substudy $(\mathrm{n}=269)$ (Table S1).

\section{The Multidomain Alzheimer Preventive Trial interventions}

The MAPT multidomain lifestyle intervention was comprised of cognitive training, nutritional counselling and physical activity counselling (26). Group-based 2-hour sessions were performed twice a week during the first four weeks of the trial, once a week for the following four weeks and then once a month for the remainder of the trial's 3-year follow-up period. The sessions comprised: one hour of cognitive training (memory and reasoning), 15 minutes of nutritional advice (based on guidelines established by the Programme National Nutrition Santé, the French National Nutrition Health Programme (27)) and 45 minutes of physical activity counselling. An exercise program was designed for each individual and participants were advised to increase the physical activity to the equivalent of at least 30 minutes walking per day 5 days a week. Two 2-hour reinforcement sessions were performed at 12 and 24 months to boost the effects of the interventions. Preventive consultations were also performed (at baseline, 12 and 24 months) with a physician to optimize the management of cardiovascular risk factors and detect functional impairments. All participants were also asked to consume two soft capsules daily as a single dose, containing either a placebo, or a total of $800 \mathrm{mg}$ of DHA and $225 \mathrm{mg}$ of EPA per day. The trial was double-blind for all subjects for n-3 PUFA supplementation or placebo allocation. No lifestyle interventions were provided to participants in the placebo alone or n-3 PUFA alone groups.

\section{$\left.{ }^{18} F\right]$ Florbetapir Positron Emission Tomography (PET)}

PET-scans as a measure of cortical $\mathrm{A} \beta$ were performed using $\left[{ }^{18} \mathrm{~F}\right]$ florbetapir as previously described (28, 29). PET data acquisitions commenced 50 minutes after injection of a mean of $4 \mathrm{MBq} / \mathrm{kg}$ weight of $\left[{ }^{18} \mathrm{~F}\right]$-Florbetapir. Radiochemical purity of $\left[{ }^{18} \mathrm{~F}\right]$-Florbetapir was superior to $99.5 \%$. Regional standard uptake value ratios (SUVRs) were generated from semi-automated quantitative analysis with the whole cerebellum used as the reference region. Cortical-to-cerebellar SUVRs (cortical-SUVRs) were obtained using the mean signal of the following predefined cortical regions: frontal, temporal, parietal, precuneus, anterior cingulate, and posterior cingulate as previously described (30). A Quality Control procedure was carried out using a semiquantification-based method. PET-scans were performed throughout the 3 year period of MAPT: the mean time of PET-scan acquisition (standard deviation, SD) was $512.7 \pm$ 249.6 days after study baseline. There was no significant difference $(p=0.223$ according to a one way analysis of variance: ANOVA) between the time interval between 
baseline and PET-scan in subjects allocated to the 4 MAPT groups (placebo: $464.9 \pm 2.62 .0$ days, $n-3$ PUFA group: $501.8 \pm 232.4$ days, multidomain + placebo: $544.4 \pm$ 237.3 days, multidomain $+n-3$ PUFA: $536.8 \pm 259.8$ days). Very few subjects were scanned before 6 months: 22 out of $269(8.2 \%)$.

\section{Covariates}

We selected the following covariates on the basis of data availability and the literature on AD (31-33): age at PET-scan assessment, gender, educational level, cognitive status assessed at the clinical visit closest to PET-scan [Clinical dementia rating (CDR): scores 0 or 0.5], time interval between baseline and PET-scan (in days), physical activity assessed at the clinical visit closest to PET-scan [measured in metabolic equivalent tasks - minutes per week (MET-min/week)] and ApoE \&4 genotype (carriers of at least one $\varepsilon 4$ allele versus noncarriers).

\section{Statistical Analysis}

Descriptive statistics are presented as mean \pm (SD) or absolute values/percentages. Clinical and demographic characteristics were compared between the participants in each group using chi squared tests for categorical variables and one-way ANOVA for continuous variables. This was a post-hoc analysis since the association between cortical A $\beta$ burden and the MAPT interventions was not an a priori hypothesis of the MAPT study. We used multiple linear regression models to compare cortical A $\beta$ levels (measured once per subject, at any time during follow-up, as described above) across the four MAPT randomization groups (placebo alone, n-3 PUFA supplementation alone, multidomain lifestyle intervention + placebo, and multidomain lifestyle intervention $+n-3$ PUFA supplementation) adjusting for all covariates. Next we dichotomized the dependent variable, cortical $\mathrm{A} \beta$, with a positivity threshold of mean cortical SUVR $\geq 1.17(28,34)$ then performed logistic regression adjusting for all covariates. We ran three sensitivity analyses in order to substantiate our main analysis. Firstly, we ran a multiple linear regression adjusting for all covariates including only those participants who had their PET-scan $\geq 12$ months postbaseline and hence had received MAPT interventions for $\geq 12$ months. Secondly, we ran a multiple linear regression adjusting for all covariates including only those participants who had their PET-scan $<12$ months post-baseline. Thirdly, we ran a multiple linear regression adjusting for all covariates after combining the MAPT data into 2 groups according to allocation to multidomain lifestyle intervention (placebo plus $n-3$ PUFA supplementation versus multidomain lifestyle intervention + placebo plus multidomain lifestyle intervention $+\mathrm{n}-3$ PUFA supplementation). To explore the role of adherence to intervention, we ran multiple linear regression restricted to subjects in the multidomain groups adjusted for age, sex and ApoE $\varepsilon 4$ carrier status to assess the association between cortical $A \beta$ and adherence to the multidomain lifestyle intervention or adherence to the multidomain lifestyle intervention $+n-3$ PUFA supplementation. Adherence was defined as $\geq 75 \%$ attendance to the sessions over the 3-year period of MAPT including participation in the two boost sessions at the 12 and 24 month follow-ups (reference: non-adherent $<75 \%$ attendance) (5). Lastly, to explore the role of time on the association of MAPT intervention with cortical $\mathrm{A} \beta$ we ran a multiple linear regression analysis (adjusted for all covariates) with the introduction of an interaction term between MAPT group allocation and time between PET-scan and baseline (in days). Due to the exploratory nature of the study there was no correction for multiple comparisons: $\mathrm{P}<0.05$ was considered statistically significant. Statistical analyses were performed using Stata version 14 (Stata Corp., College Station, TX, USA).

\section{Results}

\section{Sample characteristics}

Demographic and clinical characteristics of the participants included in this study are shown in Table 1. There were no significant between-group differences in age, gender, cognitive status, time interval between baseline and PET-scan, physical activity and number of ApoE $\varepsilon 4$ carriers. However, educational level differed significantly between groups as did cortical SUVR as a measure of $A \beta$ burden. There was less cortical SUVR present in participants receiving multidomain intervention + placebo or multidomain $+n-3$ PUFA. The mean age of the participants was approximately 76 years, and around $60 \%$ of the population were female. Participants exhibited a high level of education and almost half of the participants had a CDR score of 0.5 and approximately one third of the subjects carried at least one ApoE $\varepsilon 4$ allele.

\section{Main analysis}

In the adjusted multiple linear regression model, cortical $A \beta$ was significantly lower in the multidomain lifestyle intervention + placebo group (mean difference, $-0.088,95 \% \mathrm{CI},-0.148,-0.029, \mathrm{p}=0.004)$ and the multidomain lifestyle intervention $+\mathrm{n}-3$ PUFA group (mean difference, $-0.100,95 \% \mathrm{CI},-0.160,-0.041, \mathrm{p}=0.001$ ), compared to the placebo alone group (table 2), but there was no difference between the placebo alone and n-3 PUFA supplementation alone groups (mean difference, $-0.011,95 \% \mathrm{CI},-0.072,0.051, \mathrm{p}=0.729)$. ApoE $\varepsilon 4$ carrier status was also significantly associated with cortical $A \beta$ 
Table 1. Participant characteristics

\begin{tabular}{|c|c|c|c|c|c|}
\hline Variables & Placebo $(n=68)$ & $n-3(n=60)$ & MI + placebo $(n=68)$ & $M I+n-3(n=73)$ & P value \\
\hline Age, y & $76.07 \pm 4.78$ & $76.18 \pm 4.40$ & $76.07 \pm 4.22$ & $75.93 \pm 4.27$ & 0.991 \\
\hline Sex, women $(\%)$ & $40(58.8 \%)$ & $37(61.7 \%)$ & $43(63.2 \%)$ & $42(57.5 \%)$ & 0.900 \\
\hline No diploma or primary school certificate & $15(22.4 \%)$ & $21(35.6 \%)$ & $21(31.3 \%)$ & $11(15.3 \%)$ & \\
\hline Secondary education no high-school diploma & $18(26.9 \%)$ & $13(22.0 \%)$ & $17(25.4 \%)$ & $31(43.1 \%)$ & \\
\hline Higher diploma & $23(34.3 \%)$ & $22(37.3 \%)$ & $18(26.9 \%)$ & $16(22.2 \%)$ & \\
\hline$\%$ of CDR $0.5(\%)$ & $32(47.1 \%)$ & $28(46.7 \%)$ & $32(47.1 \%)$ & $39(53.4 \%)$ & 0.826 \\
\hline MET-min/week & $1963.21 \pm 1885.22$ & $1466.22 \pm 1360.18$ & $1759.49 \pm 1867.87$ & $1652.0 \pm 1691.85$ & 0.425 \\
\hline ApoE $\varepsilon 4$ carriers (\%) & $19(32.2 \%)$ & $13(24.1 \%)$ & $18(30.5 \%)$ & $15(23.8 \%)$ & 0.645 \\
\hline Cortical-SUVR & $1.23 \pm 0.19$ & $1.21 \pm 0.18$ & $1.13 \pm 0.15$ & $1.11 \pm 0.13$ & 0.0001 \\
\hline
\end{tabular}

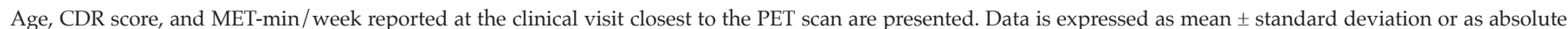

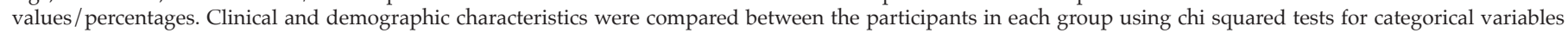

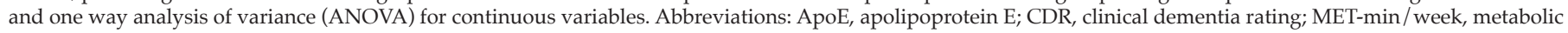
equivalent tasks - minutes per week; n-3, omega 3 polyunsaturated fatty acid supplementation; MI, multidomain intervention; SUVR, standard uptake ratio values.

Table 2. Multiple linear regressions examining the cross-sectional associations between cortical $\beta$-amyloid load and the MAPT interventions

\begin{tabular}{|l|c|c|c|c|c|c|}
\hline & \multicolumn{3}{|c|}{ Unadjusted model (n= 269) } & \multicolumn{3}{c|}{ Adjusted model (n= 231) } \\
\hline MAPT group: & B-coeff. & $\mathbf{9 5 \%}$ CI & $\mathbf{p}$ & B-coeff. & $\mathbf{9 5 \%}$ CI & p \\
\hline n-3 PUFA supplementation & -0.022 & $-0.079,0.036$ & 0.456 & -0.011 & $-0.072,0.051$ \\
\hline Multidomain + placebo & -0.095 & $-0.151,-0.040$ & 0.001 & -0.088 & $-0.148,-0.029$ \\
Multidomain + n-3 PUFA & -0.114 & $-0.169,-0.059$ & $<0.001$ & -0.100 & $-0.160,-0.041$ \\
\hline
\end{tabular}

The adjusted model contained fewer subjects due to missing data on covariates (age at PET-scan assessment, ApoE $\varepsilon 4$ genotype, gender, educational level, time interval between baseline and PET-scan, and Clinical dementia rating (CDR) and physical activity assessed at the clinical visit closest to PET-scan). B-coefficients represent the mean difference in SUVR between the placebo and intervention. Mean SUVR $(95 \% \mathrm{CI})$ for the placebo group in the unadjusted model and as predicted from the adjusted model are $1.23(1.19,1.27)$ and $1.33(0.94,1.72)$ respectively. Abbreviations: B-coeff, B-coefficient; CI, confidence intervals; n-3 PUFA, omega 3 polyunsaturated fatty acid; p, probability; SUVR, standard uptake ratio values.

in the model (mean difference, 0.118, $95 \% \mathrm{CI}, 0.071,0.166$, $\mathrm{p}<0.001$ ) with ApoE $\varepsilon 4$ carriers having greater SUVR compared to non-carriers, as expected. None of the other demographic and clinical co-variates were significantly associated with cortical $\mathrm{A} \beta$.

In the adjusted logistic regression analysis, compared to the placebo alone group, the odds of cortical amyloid positivity (defined as SUVR $\geq 1.17$ ) were significantly lower in the multidomain lifestyle intervention $+n-3$ PUFA group (odds ratio (OR), 0.31, $95 \%$ CI, 0.133,0.699, $\mathrm{p}$ $=0.005)$, but not in the multidomain lifestyle intervention + placebo group, although they were numerically lower (OR 0.61, 95 \% CI, 0.272,1.345, $\mathrm{p}=0.218$ ) (Table 3).

\section{Sensitivity analysis}

In multiple linear regression amongst participants who had their PET scans $\geq 12$ months post-baseline, and therefore had received intervention for $\geq 12$ months, results were similar to the main analysis (Table 4). Amongst participants who had their PET scans $<12$ months post-baseline cortical $A \beta$ was still significantly lower in the multidomain lifestyle intervention + n-3 group compared to the placebo alone group (B-coefficient, -0.126, $95 \% \mathrm{CI},-0.252,-0.001, \mathrm{p}=0.048$ ), but the difference between the multidomain lifestyle intervention + placebo group and the placebo alone group was not significant (B-coefficient, $-0.099,95 \%$ CI, $-0.227,0.029, \mathrm{p}=0.127$ ) (Table 5). Dividing the participants into two groups according to whether the subjects received multidomain lifestyle intervention or not gave similar results to the main analysis (B-coefficient, -0.089 , $95 \% \mathrm{CI},-0.132,-0.046, \mathrm{p}<0.001$ : reference $=$ placebo alone and n-3 PUFA supplementation alone groups combined).

\section{Exploratory analysis}

Cortical $A \beta$ was not associated with multidomain intervention adherence in the multidomain lifestyle intervention + placebo group (mean difference between adherent and non-adherent subjects , $-0.019,95 \% \mathrm{CI}$, $-0.106,0.068, p=0.668)$ nor in the multidomain lifestyle intervention + n-3 PUFA group (mean difference, 0.038, $95 \% \mathrm{CI},-0.025,0.102, \mathrm{p}=0.228)$. Furthermore, the 
Table 3. Logistic regression examining the cross-sectional associations between cortical $\beta$-amyloid and MAPT interventions

\begin{tabular}{|l|c|c|c|c|c|c|}
\hline & \multicolumn{3}{|c|}{ Unadjusted model $(\mathbf{n}=\mathbf{2 6 9})$} & \multicolumn{3}{c|}{ Adjusted model (n= 231) } \\
\hline MAPT group: & Odds ratio & $\mathbf{9 5 \%}$ CI & $\mathbf{p}$ & Odds ratio & $\mathbf{9 5 \%}$ CI & $\mathbf{p}$ \\
\hline n-3 PUFA supplementation & 0.88 & $0.437,1.753$ & 0.707 & 1.13 & $0.506,2.538$ \\
\hline Multidomain + placebo & 0.55 & $0.274,1.085$ & 0.084 & 0.61 & $0.272,1.345$ \\
\hline Multidomain + n-3 PUFA & 0.28 & $0.135,0.583$ & 0.001 & 0.31 & $0.133,0.699$ \\
\hline
\end{tabular}

$\beta$-amyloid positivity was defined with a threshold of mean cortical SUVR $\geq 1$.17. Abbreviations: CI, confidence intervals; $\mathrm{n}-3$ PUFA, omega 3 polyunsaturated fatty acid; p, probability.

Table 4. Sensitivity analysis in subjects having their PET-scan $\geq 12$ months

\begin{tabular}{|l|c|c|c|c|c|c|}
\hline & \multicolumn{3}{|c|}{ Unadjusted model (n= 182) } & \multicolumn{3}{c|}{ Adjusted model (n= 169) } \\
\hline MAPT group: & B-coeff. & $\mathbf{9 5 \%}$ CI & $\mathbf{p}$ & B-coeff. & $\mathbf{9 5 \%}$ CI & $\mathbf{p}$ \\
n-3 PUFA supplementation & -0.023 & $-0.097,0.051$ & 0.536 & -0.009 & $-0.081,0.063$ \\
\hline Multidomain + placebo & -0.083 & $-0.154,-0.012$ & 0.023 & -0.087 & $-0.157,-0.017$ & 0.015 \\
\hline Multidomain + n-3 PUFA & -0.103 & $-0.174,-0.033$ & 0.004 & -0.075 & $-0.145,-0.005$ \\
\hline
\end{tabular}

The adjusted model contained fewer subjects due to missing data on confounders. B-coefficients represent the mean difference in SUVR between the placebo and intervention. Mean SUVR (95\% CI) for the placebo group in the unadjusted model and as predicted from the adjusted model are 1.22 (1.17,1.27) and 1.28 $(0.83,1.73)$ respectively. Abbreviations: B-coeff, B-coefficient; CI, confidence intervals; n-3 PUFA, omega 3 polyunsaturated fatty acid; $p$, probability; SUVR, standard uptake ratio values.

\section{Table 5. Sensitivity analysis in subjects having their PET-scan $<12$ months}

\begin{tabular}{|l|c|c|c|c|c|c|}
\hline & \multicolumn{3}{c|}{ Unadjusted model (n= 87) } & \multicolumn{3}{c|}{ Adjusted model (n= 62) } \\
\hline MAPT group: & B-coeff. & $\mathbf{9 5 \%}$ CI & $\mathbf{p}$ & B-coeff. & $\mathbf{9 5 \%}$ CI & $\mathbf{p}$ \\
\hline n-3 PUFA supplementation & -0.009 & $-0.106,0.088$ & 0.854 & 0.047 & $-0.088,0.183$ \\
\hline Multidomain + placebo & -0.112 & $-0.214,-0.020$ & 0.019 & -0.099 & $-0.227,0.029$ & 0.127 \\
\hline Multidomain + n-3 PUFA & -0.129 & $-0.222,-0.036$ & 0.007 & -0.126 & $-0.252,-0.001$ & 0.048 \\
\hline
\end{tabular}

The adjusted model contained fewer subjects due to missing data on confounders. B-coefficients represent the mean difference in SUVR between the placebo and intervention. Mean SUVR $(95 \%$ CI) for the placebo group in the unadjusted model and as predicted from the adjusted model are 1.24 (1.18,1.30) and 1.55 $(0.63,2.48)$ respectively. Abbreviations: B-coeff, B-coefficient; CI, confidence intervals; n-3 PUFA, omega 3 polyunsaturated fatty acid; p, probability; SUVR, standard uptake ratio values.

interaction between MAPT group allocation and time between PET scan and baseline was not significantly associated with cortical $\mathrm{A} \beta$ in the model $(\mathrm{p}<0.05)$.

\section{Discussion}

We have observed that assignment to multidomain lifestyle intervention with and without n-3 PUFA supplementation were similarly associated with less cortical $A \beta$ load in older adults at risk of dementia. In contrast, n-3 PUFA supplementation alone was not associated with cortical $A \beta$. It should be noted, however that a significant association between the multidomain lifestyle intervention $+n-3$ PUFA group and cortical $A \beta$ was also observed in a sensitivity analysis restricted to those subjects who received a PET-scan $<12$ months postbaseline (although the majority of these subjects would still have received the intervention for at least 6 months prior to having their PET scan). Moreover, because it would be expected that the longer participants were exposed to the multidomain lifestyle intervention, the lower the cortical $A \beta$ burden would be, we performed an exploratory analysis for an interaction between MAPT group allocation and time between PET scan and baseline. We found no significant interaction with time. What is more, exploratory analysis showed that cortical $\mathrm{A} \beta$ was not significantly associated with adherence to the multidomain lifestyle interventions. Collectively, these findings cast some doubt on our main analysis hence further validation studies are required.

In the primary analysis of MAPT, no significant effects of any of the interventions (multidomain lifestyle intervention + placebo; n-3 PUFA supplementation; multidomain lifestyle intervention $+n-3$ PUFA supplementation) were found on a composite cognitive score, compared to placebo alone, after adjustment for multiple testing (26). However, significantly less cognitive decline during follow-up was noted in the combined intervention group and in the multidomain intervention plus placebo group than in the placebo group in the subgroup of $\mathrm{A} \beta$ positive participants $(26,35)$. These findings suggest that multidomain 
intervention might work through the reduction of cerebral $\mathrm{A} \beta$ therefore providing indirect evidence to support to the main findings of the analysis presented here. Furthermore, there is a growing body of evidence to suggest that physical activity $(4,7,8)$, cognitive activity $(1,2)$ and nutrition $(5,6)$ are independently associated with cerebral $A \beta$ levels and thus collectively these elements might offer a synergistic effect on reducing cerebral $\mathrm{A} \beta$.

In the short-term, analysis of existing longitudinal observational studies with data on cerebral $A \beta$ in which two or more components of the MAPT multidomain lifestyle intervention could be operationalized might shed more light on our preliminary findings. Whilst, in the longer-term, our study specifically begs the question 'Does multidomain lifestyle intervention reduce cortical $A \beta$ ?'. Further research in the form of a large RCT, in which cerebral $A \beta$ is measured before and after the intervention, is required to respond to this question. Establishing the correct level of multidomain lifestyle intervention also remains to be determined. In terms of physical activity and cognitive training is sustained activity or activity of increasing difficulty required? Which nutrients are more important for healthy aging, fats, specific vitamins or the correct dietary balance? Another important question to answer is: What is the best time window to administer a multi-domain intervention? Cerebral $A \beta$ accrual is believed to occur over a protracted period accounting for the long pro-dromal phase of $\mathrm{AD}$ (36); therefore, it is possible that mid-life interventions might be required to prevent future pathological changes. The duration of a lifestyle intervention is another important determinant of efficacy that requires investigation.

In conclusion we present here some evidence that multidomain lifestyle intervention both with and without n-3 PUFA supplementation were similarly associated with less cortical $A \beta$ in older adults at risk of dementia. Further validation studies are required to either support or refute our preliminary findings and to assess whether any relationships between multidomain interventions and cortical $\mathrm{A} \beta$ are causal.

\section{Conflicts of Interest: The authors declare that they have no conflict of interest.}

Sources of funding: "The MAPT study was supported by grants from the Gérontopôle of Toulouse, the French Ministry of Health (PHRC 2008, 2009), Pierre Fabre Research Institute (manufacturer of the omega-3 supplement), Exhonit Therapeutics SA, Avid Radiopharmaceuticals Inc and in part by a grant from the French National Agency for Research called "Investissements d'Avenir" n ANR11-LABX-0018-01. The promotion of this study was supported by the University Hospital Center of Toulouse. The data sharing activity was supported by the Association Monegasque pour la Recherche sur la maladie d'Alzheimer (AMPA) and the UMR 1027 Unit INSERM-University of Toulouse III".

Sponsor's role: None.

MAPT/DSA Group refers to MAPT Study Group: Principal investigator: Bruno Vellas (Toulouse); Coordination: Sophie Guyonnet ; Project leader: Isabelle Carrié CRA: Lauréane Brigitte ; Investigators: Catherine Faisant, Françoise Lala, Julien Delrieu, Hélène Villars ; Psychologists: Emeline Combrouze, Carole Badufle, Audrey Zueras ; Methodology, statistical analysis and data management: Sandrine Andrieu, Christelle Cantet, Christophe Morin; Multidomain group: Gabor Abellan
Van Kan, Charlotte Dupuy, Yves Rolland (physical and nutritional components), Céline Caillaud, Pierre-Jean Ousset (cognitive component), Françoise Lala (preventive consultation) (Toulouse). The cognitive component was designed in collaboration with Sherry Willis from the University of Seattle, and Sylvie Belleville, Brigitte Gilbert and Francine Fontaine from the University of Montreal. Co-Investigators in associated centres: Jean-François Dartigues, Isabelle Marcet, Fleur Delva, Alexandra Foubert, Sandrine Cerda (Bordeaux); Marie-NoëlleCuffi, Corinne Costes (Castres); Olivier Rouaud, Patrick Manckoundia, Valérie Quipourt, Sophie Marilier, Evelyne Franon (Dijon); Lawrence Bories, Marie-Laure Pader, Marie-France Basset, Bruno Lapoujade, Valérie Faure, Michael Li Yung Tong, Christine Malick-Loiseau, Evelyne Cazaban-Campistron (Foix); Françoise Desclaux, Colette Blatge (Lavaur); Thierry Dantoine, Cécile Laubarie-Mouret, Isabelle Saulnier, Jean-Pierre Clément, Marie-Agnès Picat, Laurence BernardBourzeix, Stéphanie Willebois, Iléana Désormais, Noëlle Cardinaud (Limoges); Marc Bonnefoy, Pierre Livet, Pascale Rebaudet, Claire Gédéon, Catherine Burdet, Flavien Terracol (Lyon), Alain Pesce, Stéphanie Roth, Sylvie Chaillou, Sandrine Louchart (Monaco); Kristelle Sudres, Nicolas Lebrun, Nadège Barro-Belaygues (Montauban); Jacques Touchon, Karim Bennys, Audrey Gabelle, Aurélia Romano, Lynda Touati, Cécilia Marelli, Cécile Pays (Montpellier); Philippe Robert, Franck Le Duff, Claire Gervais, Sébastien Gonfrier (Nice); Yannick Gasnier and Serge Bordes, Danièle Begorre, Christian Carpuat, Khaled Khales, Jean-François Lefebvre, Samira Misbah El Idrissi, Pierre Skolil, Jean-Pierre Salles (Tarbes). MRI group: Carole Dufouil (Bordeaux), Stéphane Lehéricy, Marie Chupin, JeanFrançois Mangin, Ali Bouhayia (Paris); Michèle Allard (Bordeaux); Frédéric Ricolfi (Dijon); Dominique Dubois (Foix); Marie Paule Bonceour Martel (Limoges); François Cotton (Lyon); Alain Bonafé (Montpellier); Stéphane Chanalet (Nice); Françoise Hugon (Tarbes); Fabrice Bonneville, Christophe Cognard, François Chollet (Toulouse). PET scans group: Pierre Payoux, Thierry Voisin, Julien Delrieu, Sophie Peiffer, Anne Hitzel, (Toulouse); Michèle Allard (Bordeaux); Michel Zanca (Montpellier); Jacques Monteil (Limoges); Jacques Darcourt (Nice). Medicoeconomics group: Laurent Molinier, Hélène Derumeaux, Nadège Costa (Toulouse). Biological sample collection: Bertrand Perret, Claire Vinel, Sylvie Caspar-Bauguil (Toulouse). Safety management : Pascale Olivier-Abbal. DSA Group: Sandrine Andrieu, Christelle Cantet, Nicola Coley.

Open Access: This article is distributed under the terms of the Creative Commons Attribution 4.0 International License (http://creativecommons.org/ licenses/by/4.0/), which permits use, duplication, adaptation, distribution and reproduction in any medium or format, as long as you give appropriate credit to the original author(s) and the source, provide a link to the Creative Commons license and indicate if changes were made.

\section{References}

1. S. M. Landau et al., "Association of lifetime cognitive engagement and low $\beta$-amyloid deposition," Arch. Neurol., 2012;vol. 69, no. 5, pp. 623-629, May.

2. M. Wirth, S. Villeneuve, R. La Joie, S. M. Marks, and W. J. Jagust, "Geneenvironment interactions: lifetime cognitive activity, APOE genotype, and $\beta$-amyloid burden," J. Neurosci. Off. J. Soc. Neurosci., 2014;vol. 34, no. 25, pp. 8612-8617, Jun.

3. B. M. Brown et al., "Habitual exercise levels are associated with cerebral amyloid load in presymptomatic autosomal dominant Alzheimer's disease," Alzheimers Dement. J. Alzheimers Assoc., 2017; vol. 13, no. 11, pp. 1197-1206, Nov.

4. K. Y. Liang et al., "Exercise and Alzheimer's disease biomarkers in cognitively normal older adults," Ann. Neurol., 2010 ;vol. 68, no. 3, pp. 311-318, Sep.

5. V. Berti et al., "Nutrient patterns and brain biomarkers of Alzheimer's disease in cognitively normal individuals," J. Nutr. Health Aging, 2015;vol. 19, no. 4, pp. 413-423, Apr.

6. Hooper C., De Souto Barreto P., Payoux P., Salabert A. S., Guyonnet S. Andrieu S., Vellas B, "Association of cortical B-amyloid with erythrocyte membrane monounsaturated and saturated fatty acids in older adults at risk of dementia," 2017; J. Nutr. Health Aging,

7. S. Müller et al., "Relationship between physical activity, cognition, and Alzheimer pathology in autosomal dominant Alzheimer's disease," Alzheimers Dement. J. Alzheimers Assoc., 2018;vol. 14, no. 11, pp. 1427-1437, Nov.

8. D. Head et al., "Exercise Engagement as a Moderator of the Effects of APOE Genotype on Amyloid Deposition," Arch. Neurol., 2012;vol. 69, no. 5, pp. 636-643, May.

9. B. M. Brown et al., "Physical activity and amyloid- $\beta$ plasma and brain levels: results from the Australian Imaging, Biomarkers and Lifestyle Study of Ageing," Mol. Psychiatry, 2013; vol. 18, no. 8, pp. 875-881, Aug.

10. J. Zhang, Y. Guo, Y. Wang, L. Song, R. Zhang, and Y. Du, “Long-term treadmill exercise attenuates A $\beta$ burdens and astrocyte activation in APP/ PS1 mouse model of Alzheimer's disease," Neurosci. Lett., 2017;vol. 666, pp. 70-77, Dec

11. K. A. Alkadhi and A. T. Dao, "Exercise decreases BACE and APP levels in the hippocampus of a rat model of Alzheimer's disease," Mol. Cell. Neurosci., 2018;vol. 86, pp. 25-29, Jan.

12. P. Vemuri et al., "Effect of intellectual enrichment on AD biomarker 
trajectories: Longitudinal imaging study," Neurology, 2016;vol. 86, no. 12, pp. 1128-1135, Mar.

13. J. R. Cirrito et al., "Synaptic activity regulates interstitial fluid amyloid-beta levels in vivo," Neuron, 2005;vol. 48, no. 6, pp. 913-922, Dec. .

14. A. W. Bero et al., "Neuronal activity regulates the regional vulnerability to amyloid- $\beta$ deposition," Nat. Neurosci., 2011;vol. 14, no. 6, pp. 750-756, Jun. .

15. O. Lazarov et al., "Environmental enrichment reduces Abeta levels and amyloid deposition in transgenic mice," Cell, 2005;vol. 120, no. 5, pp. 701-713, Mar. .

16. M. K. Taylor et al., "A high-glycemic diet is associated with cerebral amyloid burden in cognitively normal older adults," Am. J. Clin. Nutr., 2017;vol. 106, no. 6 , pp. $1463-1470$, Dec. .

17. V. Berti et al., "Mediterranean diet and 3-year Alzheimer brain biomarker changes in middle-aged adults," Neurology, 2018;Apr.

18. M. O. W. Grimm et al., "Docosahexaenoic acid reduces amyloid beta production via multiple pleiotropic mechanisms," J. Biol. Chem., 2011;vol. 286, no. 16, pp. 14028-14039, Apr.

19. S. E. Perez et al., "DHA diet reduces AD pathology in young APPswe/PS1 Delta E9 transgenic mice: possible gender effects," J. Neurosci. Res., 2010;vol. 88, no. 5, pp. 1026-1040, Apr.

20. G. P. Lim et al., "A diet enriched with the omega-3 fatty acid docosahexaenoic acid reduces amyloid burden in an aged Alzheimer mouse model," J. Neurosci. Off. J. Soc. Neurosci., 2005;vol. 25, no. 12, pp. 3032-3040, Mar.

21. H. N. Yassine et al., "Association of Serum Docosahexaenoic Acid With Cerebral Amyloidosis," JAMA Neurol., 2016;Aug.

22. C. Hooper et al., "Cross-sectional associations of cortical $\beta$-amyloid with erythrocyte membrane long-chain polyunsaturated fatty acids in older adults with subjective memory complaints," J. Neurochem., 2017;May.

23. T. Ngandu et al., "A 2 year multidomain intervention of diet, exercise, cognitive training, and vascular risk monitoring versus control to prevent cognitive decline in at-risk elderly people (FINGER): a randomised controlled trial," Lancet Lond. Engl., 2015; vol. 385, no. 9984, pp. 2255-2263, Jun.

24. D. E. Barnes et al., "The Mental Activity and eXercise (MAX) trial: a randomized controlled trial to enhance cognitive function in older adults," JAMA Intern. Med., 2013; vol. 173, no. 9, pp. 797-804, May

25. E. P. Moll van Charante et al., "Effectiveness of a 6-year multidomain vascular care intervention to prevent dementia (preDIVA): a cluster-randomised controlled trial," Lancet Lond. Engl., 2016;vol. 388, no. 10046, pp. 797-805, Aug.
26. S. Andrieu et al., "Effect of long-term omega 3 polyunsaturated fatty acid supplementation with or without multidomain intervention on cognitive function in elderly adults with memory complaints (MAPT): a randomised, placebo-controlled trial," Lancet Neurol., 2017;Mar.

27. S. Hercberg, S. Chat-Yung, and M. Chaulia, "The French National Nutrition and Health Program: 2001-2006-2010," Int. J. Public Health, 2008;vol. 53, no. 2, pp. 68-77.

28. N. Del Campo et al., "Relationship of regional brain $\beta$-amyloid to gait speed," Neurology, 2016 ;vol. 86, no. 1, pp. 36-43, Jan.

29. B. Vellas et al., "MAPT STUDY: A MULTIDOMAIN APPROACH FOR PREVENTING ALZHEIMER'S DISEASE: DESIGN AND BASELINE DATA," J. Prev. Alzheimers Dis., 2014;vol. 1, no. 1, pp. 13-22, Jun.

30. A. D. Joshi et al., "Performance characteristics of amyloid PET with florbetapir F 18 in patients with alzheimer's disease and cognitively normal subjects," J. Nucl. Med. Off. Publ. Soc. Nucl. Med., 2012;vol. 53, no. 3, pp. 378-384, Mar.

31. Alzheimer's Association, "2016 Alzheimer's disease facts and figures," Alzheimers Dement. J. Alzheimers Assoc., 2016;vol. 12, no. 4, pp. 459-509, Apr.

32. P. de S. Barreto et al., "Effects of multidomain lifestyle intervention, omega3 supplementation or their combination on physical activity levels in older adults: secondary analysis of the Multidomain Alzheimer Preventive Trial (MAPT) randomised controlled trial," Age Ageing, pp. 1-8, Nov. 2017.

33. P. de Souto Barreto et al., "Physical Activity and Amyloid- $\beta$ Brain Levels in Elderly Adults with Intact Cognition and Mild Cognitive Impairment," J. Am. Geriatr. Soc., vol. 63, no. 8, pp. 1634-1639, Aug. 2015.

34. A. S. Fleisher et al., "Using positron emission tomography and florbetapir F18 to image cortical amyloid in patients with mild cognitive impairment or dementia due to Alzheimer disease," Arch. Neurol., 2011;vol. 68, no. 11, pp. 1404-1411, Nov.

35. J. Delrieu et al., "Multidomain intervention and/or omega-3 in nondemented elderly subjects according to amyloid status," Alzheimers Dement. J. Alzheimers Assoc., 2019;Sep.

36. V. L. Villemagne et al., "Amyloid $\beta$ deposition, neurodegeneration, and cognitive decline in sporadic Alzheimer's disease: a prospective cohort study," Lancet Neurol., 2013;vol. 12, no. 4, pp. 357-367, Apr. 